\title{
Yves Meyer Awarded Abel Prize
}

The Norwegian Academy of Science and Letters has awarded the 2017 Abel Prize to YVES MEYeR of École Normale Supérieure Paris-Saclay, France, "for his pivotal role in the development of the mathematical theory of wavelets." The Abel Prize recognizes contributions of extraordinary depth and influence to the mathematical sciences and has been awarded annually since 2003. It carries a cash award of 6,000,000 Norwegian krone (approximately US $\$ 715,000$ ).

\section{Citation}

Fourier analysis provides a useful way of decomposing a signal or function into simply-structured pieces such as sine and cosine waves. These pieces have a concentrated frequency spectrum but are very spread out in space. Wavelet analysis provides a way of cutting up functions into pieces that are localized in both frequency and space. Yves Meyer was the visionary leader in the modern development of this theory, at the intersection of mathematics, information technology, and computational science.

The history of wavelets goes back over a hundred years, to an early construction by Alfréd Haar. In the late 1970s the seismologist Jean Morlet analyzed reflection data obtained for oil prospecting and empirically introduced a new class of functions, now called "ondelettes" or "wavelets," obtained by both dilating and translating a fixed function.

In the spring of 1985, Yves Meyer recognized that a recovery formula found by Morlet and Alex Grossmann was an identity previously discovered by Alberto Calderón. At that time, Yves Meyer was already a leading figure in the Calderón-Zygmund theory of singular integral operators. Thus began Meyer's study of wavelets, which in less than ten years would develop into a coherent and widely applicable theory.

The first crucial contribution by Meyer was the construction of a smooth orthonormal wavelet basis. The existence of such a basis had been in doubt. As in Morlet's construction, all of the functions in Meyer's basis arise by translating and dilating a single smooth "mother wavelet," which can be specified quite explicitly. Its construction, though essentially elementary, appears rather miraculous.

For permission to reprint this article, please contact:

reprint-permission@ams.org.

DOI: http://dx.doi.org/10.1090/noti1543

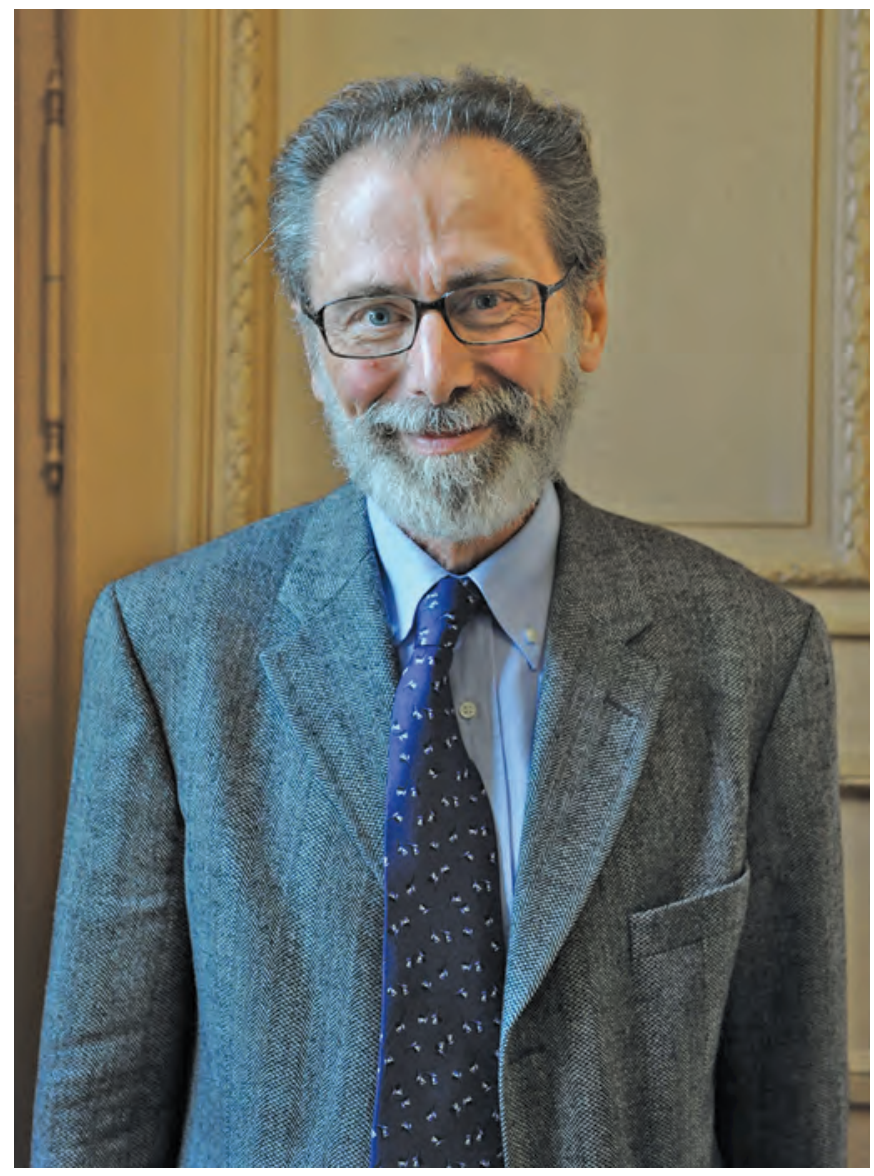

Yves Meyer

Stéphane Mallat and Yves Meyer then systematically developed multiresolution analysis, a flexible and general framework for constructing wavelet bases, which places many of the earlier constructions on a more conceptual footing. Roughly speaking, multiresolution analysis allows one to explicitly construct an orthonormal wavelet basis from any bi-infinite sequence of nested subspaces of $L^{2}(R)$ that satisfy a few additional invariance properties. This work paved the way for the construction by Ingrid Daubechies of orthonormal bases of compactly supported wavelets.

In the following decades, wavelet analysis has been applied in a wide variety of arenas as diverse as applied and computational harmonic analysis, data compression, noise reduction, medical imaging, archiving, digital cinema, 
deconvolution of the Hubble space telescope images, and the recent LIGO detection of gravitational waves created by the collision of two black holes.

Yves Meyer has also made fundamental contributions to problems in number theory, harmonic analysis, and partial differential equations, on topics such as quasicrystals, singular integral operators, and the Navier-Stokes equations. The crowning achievement of his pre-wavelets work is his proof, with Ronald Coifman and Alan McIntosh, of the $L^{2}$-boundedness of the Cauchy integral on Lipschitz curves, thus resolving the major open question in Calderón's program. The methods developed by Meyer have had a long-lasting impact in both harmonic analysis and partial differential equations. Moreover, it was Meyer's expertise in the mathematics of the Calderón-Zygmund school that opened the way for the development of wavelet theory, providing a remarkably fruitful link between a problem set squarely in pure mathematics and a theory with wide applicability in the real world.

\section{Biographical Sketch}

Yves Meyer, professor emeritus at the École Normale Supérieure Paris-Saclay in France, proves that, in contrast to what F. Scott Fitzgerald said about American lives, in mathematics a life can indeed have a second act, and perhaps even several more. Having made important contributions in the field of number theory early in his career, Meyer's boundless energy and curiosity prompted him to work on methods for breaking down complex mathematical objects into simpler wavelike components-a topic called harmonic analysis. This led him, in turn, to help construct a theory for analyzing complicated signals, with important ramifications for computer and information technologies. Then he moved on again to tackle fundamental problems in the mathematics of fluid flow.

That tendency to cross boundaries was with him from the start. Born on July 19, 1939, of French nationality, he grew up in Tunis on the North African coast. "The Tunis of my childhood was a melting pot where people from all over the Mediterranean had found sanctuary," he said in a 2011 interview. "As a child I was obsessed by the desire of crossing the frontiers between these distinct ethnic groups."

Meyer entered the elite École Normale Supérieure de la rue d'Ulm in Paris in 1957, coming in first in the entrance examination. "If you enter ENS-Ulm, you know that you are giving up money and power," he later said. "It is a choice of life. Your life will be devoted to acquiring and transmitting knowledge."

After graduating, Meyer completed his military service as a teacher in a military school. But despite his deep commitment to education and his students, he wasn't suited to the role. "A good teacher needs to be much more methodical and organized than I was," he admits. Moreover, he was uncomfortable with being the one who was "always right." "To do research," Meyer has said, "is to be ignorant most of the time and often to make mistakes." Nevertheless, he feels his experience of high school teaching shaped his life: "I understood that I was more happy to share than to possess."

He joined the University of Strasbourg as a teaching assistant, and in 1966 he was awarded a PhD there-officially under Jean-Pierre Kahane, but Meyer asserts that, like some others in France at that time, he essentially supervised himself. He became a professor of mathematics first at the Université Paris-Sud (as it is now known), then the École Polytechnique and the Université Paris-Dauphine. He moved to the ENS Cachan (recently renamed the ENS Paris-Saclay) in 1995, where he worked at the Centre of Mathematics and Its Applications (CMLA) until formally retiring in 2008. He is still an associate member of the research centre.

Yves Meyer's work has, in the most general terms, been concerned with understanding mathematical functions with complex and changing forms: a character that can be described by so-called partial differential equations. Fluid flow, for example, is described by a set of such equations called the Navier-Stokes equations, and in the 1990s Meyer helped to elucidate particular solutions to them-a topic that ranks among the biggest challenges in mathematics.

Meyer's interest in what might be called the structures and regularities of complicated mathematical objects led him in the 1960s to a theory of "model sets": a means of describing arrays of objects that lack the perfect regularity and symmetry of crystal lattices. This work, which arose from number theory, provided the underpinning theory for materials called quasicrystals, first identified in metal alloys in 1982 but prefigured by quasiregular tiling schemes identified by mathematical physicist Roger Penrose in 1974. The discovery of quasicrystals by materials scientist Dan Shechtman earned him the 2011 Nobel Prize in chemistry. Meyer has sustained his interest in quasicrystals, and, together with Basarab Matei, in 2010 he helped to elucidate their mathematical structure.

In the 1970s Meyer made profound contributions to the field of harmonic analysis, which seeks to decompose complex functions and signals into components made of simple waves. Along with Ronald Coifman and Alan McIntosh, he solved a long-standing problem in the field in 1982 by proving a theorem about a construction called the Cauchy integral operator. This interest in harmonic decomposition led Meyer into wavelet theory, which enables complex signals to be "atomized" into a kind of mathematical particle called a wavelet.

Wavelet theory began with the work of, among others, physics Nobel laureates Eugene Wigner and Dennis Gabor, geophysicist Jean Morlet, theoretical physicist Alex Grossmann, and mathematician Jan-Olov Strömberg. During a conversation over the photocopier at the Ecole Polytechnique in 1984, Meyer was handed a paper on the subject by Grossmann and Morlet and was captivated. "I took the first train to Marseilles, where I met Ingrid Daubechies, Alex Grossmann, and Jean Morlet," he says. "It was like a fairy tale. I felt I had finally found my home."

From the mid-1980s, in what he called a "second scientific life," Meyer, together with Daubechies and Coifman, brought together earlier work on wavelets into a unified 
picture. In particular, Meyer showed how to relate Grossmann and Morlet's wavelets to the work of Argentinian mathematician Alberto Calderón, which had supplied the basis for some of Meyer's most significant contributions to harmonic analysis. In 1986 Meyer and Pierre Gilles Lemarié-Rieusset showed that wavelets may form mutually independent sets of mathematical objects called orthogonal bases.

Coifman, Daubechies, and Stéphane Mallat went on to develop applications to many problems in signal and image processing. Wavelet theory is now omnipresent in many such technologies. Wavelet analysis of images and sounds allows them to be broken down into mathematical fragments that capture the irregularities of the pattern using smooth, "well-behaved" mathematical functions. This decomposition is important for image compression in computer science, being used, for example, in the JPEG 2000 format. Wavelets are also useful for characterizing objects with very complex shapes, such as so-called multifractals, and Meyer says that they prompted his interest in the Navier-Stokes equations in the mid-1990s.

In the past twenty years Meyer's passion for the structure of oscillating patterns has led him to contribute to the success of the Herschel deep-space telescope mission, and he is working on algorithms to detect cosmic gravitational waves. Meyer's contribution to image processing is also wide ranging. In 2001 he proposed a mathematical theory to decompose any image into a "cartoon" and a "texture." This "cartoon plus texture" algorithm is now routinely used in criminal investigations to extract digital fingerprints from a complex background.

In such ways, Meyer's work has a relevance extending from theoretical areas of mathematics such as harmonic analysis to the development of practical tools in computer and information science. As such, it is a perfect example of the claim that work in pure mathematics often turns out to have important and useful real-world applications.

Meyer is a member of the French Academy of Science and an honorary member of the American Academy of Arts and Sciences. He was elected a Fellow of the AMS in 2012. His previous prizes include the Salem Prize (1970) and the Gauss Prize (2010), the latter awarded jointly by the International Mathematical Union and the German Mathematical Society for advances in mathematics that have had an impact outside the field. The diversity of his work, reflected in its broad range of application, reflects his conviction that intellectual vitality is kept alive by facing fresh challenges. He has been quoted as saying that when you become too much an expert in a field, then you should leave it-but he is wary of sounding arrogant here. "I am not smarter than my more stable colleagues," he says simply. "I have always been a nomad-intellectually and institutionally."

Some feel that Meyer has not yet had the recognition his profound achievements warrant, perhaps because he has been so selfless in promoting the careers of others and in devoting himself to mathematical education as well as research. "The progress of mathematics is a collective enterprise," he has said. "All of us are needed."
He has inspired a generation of mathematicians who have gone on to make important contributions in their own right. His collaborator on wavelet theory Stéphane Mallat calls him a "visionary" whose work cannot be labeled either pure or applied mathematics, nor computer science, either, but simply "amazing." His students and colleagues speak of his insatiable curiosity, energy, generosity, and openness to other fields. "You must dig deeply into your own self in order to do something as difficult as research in mathematics," Meyer claims. "You need to believe that you possess a treasure hidden in the depths of your mind, a treasure which has to be unveiled."

AMS President Kenneth A. Ribet said, "On behalf of the American Mathematical Society, it is my great pleasure to congratulate Professor Yves Meyer, recipient of the 2017 Abel Prize. Professor Meyer has been a visionary in a broad range of fields, including number theory and differential equations. His fundamental work in the theory of wavelets has transformed the world of signal processing and has led to a myriad of practical applications."

\section{About the Prize}

The Niels Henrik Abel Memorial Fund was established in 2002 to award the Abel Prize for outstanding scientific work in the field of mathematics. The prize is awarded by the Norwegian Academy of Science and Letters, and the choice of Abel Laureate is based on the recommendation of the Abel Committee, which consists of five internationally recognized research scientists in the field of mathematics. The Committee is appointed for a period of two years. The members of the current committee are:

- John Rognes (Chair)

- Marta Sanz-Solé

- Luigi Ambrosio

- Marie-France Vignéras

- Ben J. Green

Previous recipients of the Abel Prize are:

- Jean-Pierre Serre (2003)

- Michael Atiyah and I. M. Singer (2004)

- Peter Lax (2005)

- Lennart Carleson (2006)

- S. R. S. Varadhan (2007)

- John G. Thompson and Jacques Tits (2008)

- Mikhail L. Gromov (2009)

- John Tate (2010)

- John Milnor (2011)

- Endre Szemerédi (2012)

- Pierre Deligne (2013)

- Yakov Sinai (2014)

- John F. Nash Jr. and Louis Nirenberg (2015)

- Andrew J. Wiles (2016)

\section{-From an announcement of the Norwegian Academy of Science and Letters}

Photo Credit

The photo of Yves Meyer is $\odot$ B. Eymann-Académie des sciences. 